\title{
First Muon RF Acceleration for the Muon g-2 Experiment at J-PARC
}

\section{Masashi Otani*}

High Energy Accelerator Research Organization (KEK), Tsukuba, Ibaraki 305-0801, Japan

E-mail: masashio@post.kek.jp

\begin{abstract}
Muons have been accelerated by using a radio frequency accelerator for the first time. Negative muonium atoms $\left(\mathrm{Mu}^{-}\right)$, which are bound states of positive muons $\left(\mu^{+}\right)$and two electrons, are generated from $\mu^{+}$'s through the electron capture process in an aluminum degrader. The generated $\mathrm{Mu}^{-}$'s are initially electrostatically accelerated and injected into a radio frequency quadrupole linac (RFQ). In the RFQ, the $\mathrm{Mu}^{-}$'s are accelerated to $89 \mathrm{keV}$. The accelerated $\mathrm{Mu}^{-}$'s are identified by momentum measurement and time of flight. This first muon acceleration is one of big milestones for realizing new muon $\mathrm{g}$-2 experiment at J-PARC.
\end{abstract}

EPS-HEP 2017, European Physical Society conference on High Energy Physics 5-12 July 2017

Venice, Italy

${ }^{*}$ Speaker. 


\section{Introduction}

Muon acceleration using radio-frequency (RF) accelerator has been discussed for its potential advantages. For example, in muon collider and neutrino factory studies [1], it is proposed that the large transverse emittance of the muon beam can be reduced through ionisation energy loss and subsequent acceleration [2]. In material and life sciences, one promising application of muon acceleration is in the construction of a transmission muon microscope. If the muons can be cooled to the thermal temperature (ultraslow muon, USM) and subsequently re-accelerated, transmission muon microscopes will be realized [3].

Another application of USM acceleration is precise measurement of the muon anomalous magnetic moment $a_{\mu}=(g-2)_{\mu} / 2$ and electric dipole moment (EDM) at J-PARC [4]. Muon acceleration is essential to realize these applications; however, it has not been demonstrated except for simple electrostatic acceleration. In October 2017, we successfully demonstrated the first negative muonium ions $\left(\mathrm{Mu}^{-}\right.$'s) [5], which will be reported in this paper.

\section{Muon g-2 Experiment at J-PARC}

Though the discovery of Higgs at LHC completed a list of the particles predicted in Standard Model (SM) of elementary particle physics, some observations such as possible existence of dark matter indicate new physics beyond SM at some energy or interaction scale. One of the clues for new physics is the muon anomalous magnetic moment $a_{\mu}$; it has been measured to $0.54 \mathrm{ppm}$ [6] and a discrepancy of more than 3 standard deviations between measurement and the SM prediction was observed. This anomaly stimulated theorists to performing challenging calculations of corrections and uncertainties in the SM prediction of $a_{\mu}$. After many years of scrutiny, the discrepancy remains unexplained [7]. Along with the theoretical efforts, new experiments should address this anomaly.

The previous experiment at Brookhaven National Laboratory (BNL), E821, used the technique so called magic momentum. Because the muon beam generated from the secondary pions has large emittance, strong electric focusing in addition to the magnetic field is necessary in a storage ring. The difference of the cyclotron motion frequency $\left(\vec{\omega}_{c}\right)$ and the muon spin precession frequency $\left(\vec{\omega}_{s}\right)$ is given by

$$
\vec{\omega}_{a}=\vec{\omega}_{s}-\vec{\omega}_{c}=-\frac{e}{m}\left[a_{\mu} \vec{B}-\left(a_{\mu}-\frac{1}{\gamma^{2}-1}\right) \frac{\vec{\beta} \times \vec{E}}{c}+\frac{\eta}{2}\left(\vec{\beta} \times \vec{B}+\frac{\vec{E}}{c}\right)\right],
$$

where $e$ is elementary charge, $m$ is muon mass, $a_{\mu}$ is anomalous magnetic moment, $\gamma$ is the Lorentz factor, $\beta$ is the ratio of particle velocity to the speed of light $c$, and $\eta$ is electric dipole moment. The second term depending on the electric field is eliminated when the muon momentum is 3.094 $\mathrm{GeV} / \mathrm{c}$, so called magic momentum. Measurement using a new method will verify the $a_{\mu}$ anomaly.

The J-PARC E34 experiment [4] aims to measure $a_{\mu}$ with a precision of $0.1 \mathrm{ppm}$ and search for EDM with a sensitivity of about $10^{-21} e \cdot \mathrm{cm}$ by utilizing USM. Figure 1 shows the experimental setup. The experiment utilizes the proton beam from the $3 \mathrm{GeV}$ synchrotron ring to Material and Life Science Experiment Facility (MLF). The proton beam is injected to the graphite target. The generated surface muons are extracted to one of the muon beamline, H-line. The surface muons 
stop in the silica aerogel and approximately half of the stopped muons form thermal muoniums $\left(\mu^{+} e^{-}\right)$. The paired electron in the muonium is knocked out by a laser and thermal muon (3 $\mathrm{keV} / \mathrm{c}$ ) is generated. Then the muon is accelerated up to $300 \mathrm{MeV} / \mathrm{c}$ and injected to the storage ring supplying $3 \mathrm{~T}$. The decay positron is detected by a silicon strip tracker and the spin precession frequency is obtained from variation of counting rate of the decay positron. Thanks to the ultracold beam $\left(\sigma_{p T} / p=10^{-5}\right)$ where $p_{T}$ is the transverse momentum of the beam particles, the electric focusing is not necessary anymore. Eq. 2.1 becomes

$$
\vec{\omega}_{a}=-\frac{e}{m}\left[a_{\mu} \vec{B}+\frac{\eta}{2}(\vec{\beta} \times \vec{B})\right] .
$$

The anomalous magnetic moment and EDM are perpendicular to each other. Therefore these can be measured simultaneously.

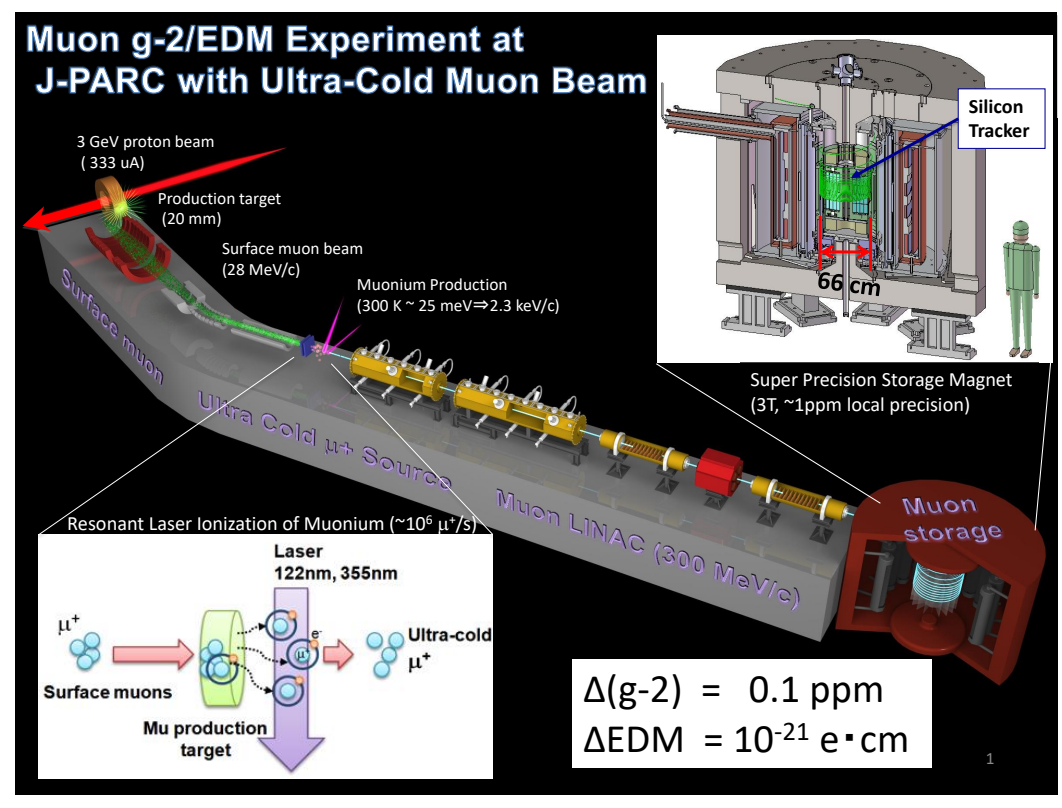

Figure 1: Overview of the J-PARC E34 experiment.

One of milestones for the experiment is demonstration of muon acceleration using a RF accelerator, which had been realized in the world.

\section{Setup of the Muon Acceleration Experiment}

Figure 2 shows the setup of the muon acceleration experiment. The J-PARC muon science facility (MUSE) [8] provides a pulsed surface muon $\left(\mu^{+}\right)$beam with the $25-\mathrm{Hz}$ repetition rate. The surface muons are decelerated by an aluminum degrader, and some portions form the negative muonium $\left(\mathrm{Mu}^{-}, \mu^{+} e^{-} e^{-}\right)$. The $\mathrm{Mu}^{-}$'s are extracted and accelerated to $5.6 \mathrm{keV}$ by an electrostatic lens [9]. They are then injected to an RFQ and accelerated to $89 \mathrm{keV}$. The accelerated $\mathrm{Mu}^{-}$'s are detected by a beam profile monitor (BPM) after a diagnostic beamline. The diagnostic beamline consists of a magnetic quadrupole pair (QM1 and QM2) and a bending magnet. 


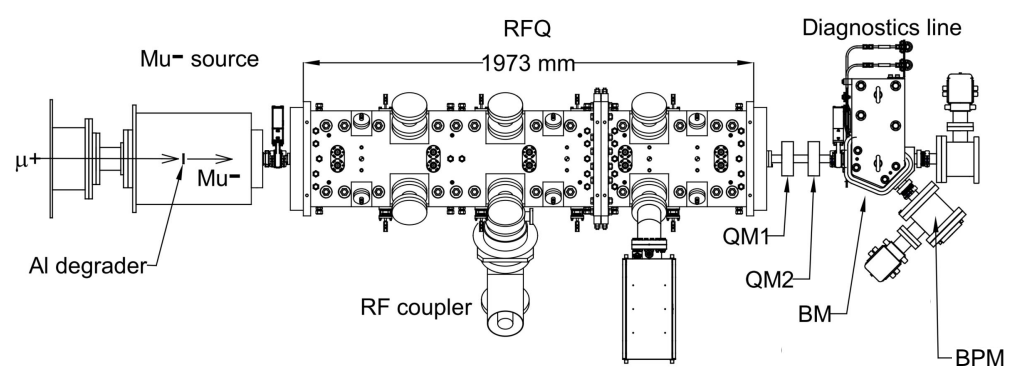

Figure 2: Schematic drawing of the experimental setup [5].

The diagnostic beamline was commissioned prior to the experiment using a $\mathrm{H}^{-}$source [10]: The $\mathrm{H}^{-}$'s are generated by exposing an $\mathrm{Al}$ foil surface to ultraviolet light. The extracted kinetic energy of the $\mathrm{H}^{-}$'s is set to $10 \mathrm{keV}$ so that the momentum of the $\mathrm{H}^{-}$'s is the same as that of the accelerated $\mathrm{Mu}^{-}$'s.

\section{Result}

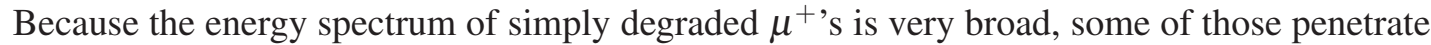
the RFQ without acceleration. The beam diagnostics system was verified using this penetrating $\mu^{+}$ at the beginning of the muon acceleration experiment. Figure 3(A) is a scatter plot of pulse height vs. time of flight (TOF) for the observed $\mu^{+}$with the MCP. The muon arrival time at the Al degrader was measured with a set of scintillating counters located at the side of the Al degrader. Figure 3(B) is a projection to the pulse-height axis. The main background of the muon measurement is decay positrons from the $\mu^{+}$. They penetrate the MCP, and thus are easily eliminated by applying a pulseheight cut. Figure 3(C) shows the TOF distribution after the pulse-height cut was applied. Because the distance between the $\mathrm{Al}$ degrader and the MCP is $3.4 \mathrm{~m}$, the TOF of the $89 \mathrm{keV} \mu^{+}$is $270 \mathrm{~ns}$. The observed TOF peak is consistent with this calculation.

Finally, the polarities of the magnets were flipped to the negative-charge configuration. Figure 4 shows the TOF spectrum with and without the RF operation after the pulse-height cut was applied. With the RF operation, a clear peak was observed at $830 \pm 11 \mathrm{~ns}$. The number of cells of this $324-\mathrm{MHz}$ RFQ is 297 , and thus it takes $\frac{297}{2 \times 324 \times 10^{6}}=458 \mathrm{~ns}$ to fully accelerate the particles through the $324 \mathrm{MHz} \mathrm{RFQ}$. Therefore, the arrival time of the accelerated $\mathrm{Mu}^{-}$is later than that of the penetrated $\mu^{+}$. The TOF spectrum was confirmed with a series of simulations. The simulation of the Soa lens was conducted using GEANT4. The three-dimensional electric field was calculated with OPERA3D [11] and implemented in the simulation. The transit time through the Soa lens was estimated with this simulation to be $307 \mathrm{~ns}$ and the acceptance was estimated to be 4\%. PARMTEQM [12] was employed for the RFQ simulation, and the transmission was estimated to be $5 \%$. Almost all losses occurred at the RFQ entrance, because of much larger emittances than the acceptance of the RFQ. TRACE3D [13] and PARMILA [14] were utilized for the diagnostics line simulation. The transport efficiency to the MCP was evaluated to be $87 \%$. The length of the diagnostics line is $0.91 \mathrm{~m}$, and thus the transit time of the $89 \mathrm{keV} \mathrm{Mu}^{-}$is $72 \mathrm{~ns}$. The total flight time of the accelerated $\mathrm{Mu}^{-}$from the $\mathrm{Al}$ degrader to the MCP was calculated to be 


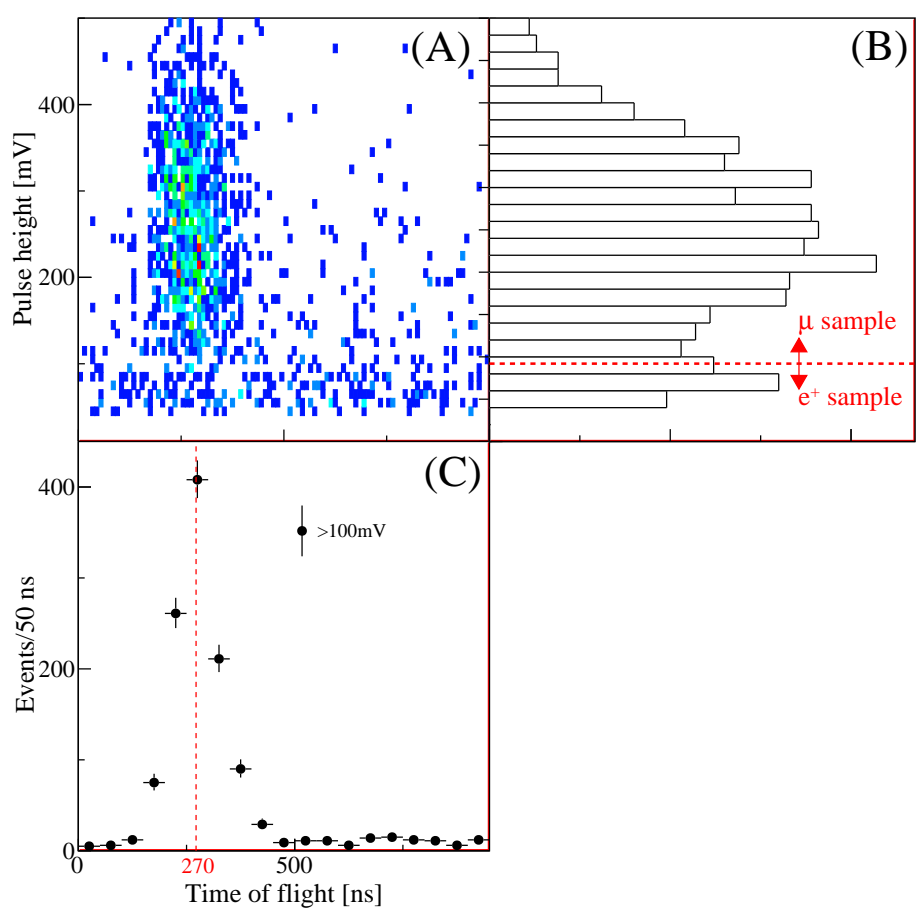

Figure 3: Distribution of the MCP pulse height and the TOF of the penetrating $\mu^{+}$[5]. (A) Scatter plot of the pulse height vs TOF. (B) Pulse height of the MCP signal. The events above $100 \mathrm{mV}$ were regarded as $\mu^{+}$. (C) TOF spectrum after the pulse-height cut was applied. The peak corresponds to the $\mu^{+}$'s injected into the RFQ with an energy of $89 \mathrm{keV}$.

$t_{\text {tran. }}=307+458+72=837 \mathrm{~ns}$, which is consistent with the measurement. The hatched histogram in Fig. 4 represents the simulated TOF spectrum of the accelerated $\mathrm{Mu}^{-}$. The number of simulation events was normalized to $4 \times 10^{11}$ incident $\mu^{+}$'s. The $46 \mathrm{~ns}$ rms width of the TOF spectrum is consistent with that from the timing distribution of the primary $\mu^{+}$at the $\mathrm{Al}$ degrader.

From these experimental results, it is concluded that the observed TOF peak is due to the $\mathrm{Mu}^{-}$'s accelerated by the RFQ to $89 \mathrm{keV}$. The event rate in the 780 to $980 \mathrm{~ns}$ TOF range was estimated to be $(5 \pm 1) \times 10^{-4} / \mathrm{s}$ by subtracting the decay-positron events estimated from the timing region outside the signal range.

\section{Summary}

In summary, muons have been accelerated by RF acceleration for the first time. Slow $\mathrm{Mu}^{-}$'s were generated through the electron capture process of the degraded $\mu^{+}$'s in the D2 area of J-PARC MUSE, and accelerated with the RFQ up to $89 \mathrm{keV}$. The intensity of the accelerated $\mathrm{Mu}^{-}$in this experiment is limited by the very low conversion efficiency of $\mu^{+}$to $\mathrm{Mu}^{-}$. With the construction of the new $\mathrm{H}$ line and the laser-dissociation ultraslow muon source, the intensity is expected to be $10^{6} / \mathrm{s}$, The result presented in this letter is the first step toward making the low-emittance muon beam available as a powerful tool for application in material and life sciences and fundamental physics research. 


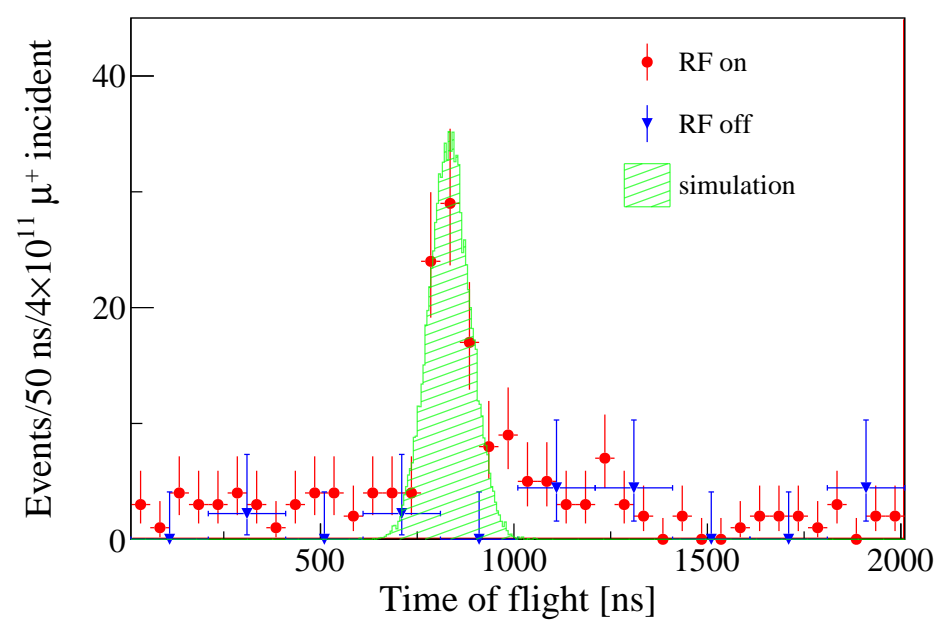

Figure 4: TOF spectra of the negative-charge configuration with RF on and off [5]. The clear peak of the $\mathrm{RF}$ on spectrum at $830 \mathrm{~ns}$ corresponds to the accelerated $\mathrm{Mu}^{-}$'s. The error bars are statistical. A simulated TOF spectrum of the accelerated $\mathrm{Mu}^{-}$'s is also plotted.

\section{Acknowledgement}

This work is supported by JSPS KAKENHI Grant Numbers , JP15H03666, JP15H05742, JP16H03987, JP16J07784, and 18H03707.

\section{References}

[1] M.A. Palmer, ICFA Beam Dynamics Newsletter, 55 (ICFA, 2011).

[2] D. Neuffer, Part. Accel. 14, 75 (1983).

[3] http://slowmuon.kek.jp/MuonMicroscopy_e.html

[4] http://g-2.kek.jp/portal/index.html

[5] S. Bae et al., Phys. Rev. Accel. Beams 21 (2018) 050101

[6] G.W. Bennett et al.: Phys. Rev. D 73 (2006) 072003

[7] A. Keshavarzi et al.: Phys. Rev. D 97 (2018) 114025

[8] Y. Miyake et al., Journal of Physics: Conference Series 225, 012036 (2010).

[9] K. F. Canter et al., in "Positron studies of solids, surfaces and atom" (World Scientific, Singapore, 1986) p. 199.

[10] Y. Nakazawa et al in Proceedings of IPAC2018 (Vancouver Canada, 2006) pp. 997 - 1000.

[11] OPERA3D, Vector Fields Limited, Oxford, England, https ://operafea.com/

[12] K. R. Crandall et al., âĂİRFQ Design CodesâĂİ, LA-UR-96-1836 (1996).

[13] K. R. Crandall and D. P. Rusthoi, LA-UR-97-886 (1997).

[14] H. Takeda, LA-UR-98-4478 (1998). 